\title{
Essentials of a Theory for How Brain Structure Contributes to the Substance of Consciousness
}

\author{
Eric Bond \\ Whitman College, Walla Walla, WA, USA \\ Email: enrique5043@outlook.com
}

How to cite this paper: Bond, E. (2022) Essentials of a Theory for How Brain Structure Contributes to the Substance of Consciousness. World Journal of Neuroscience, $12,8-21$.

https://doi.org/10.4236/wjns.2022.121002

Received: December 22, 2021

Accepted: February 6, 2022

Published: February 9, 2022

Copyright (อ 2022 by author(s) and Scientific Research Publishing Inc. This work is licensed under the Creative Commons Attribution International License (CC BY 4.0).

http://creativecommons.org/licenses/by/4.0/ (c) (i) Open Access

\begin{abstract}
Neuroscience and physics have progressed far enough that the explanatory gap between models of matter and the substance of perceptual experience is tantalizingly close to being bridged, at least insofar as consciousness is produced by the brain. This paper aims to describe the basics of how signals are transmitted within neurons via electromagnetic energy fluctuations, how EM fields emergent from these energy flows manifest as the subconscious and an experience of willed agency, as well as how the quantum principles which both EM radiation and atomic structure abide combine them to form percepts from electromagnetic matter. This might be the most promising option yet for fashioning a physical paradigm that theorizes consciousness.
\end{abstract}

\section{Keywords}

Consciousness, Electromagnetic Field, Electromagnetic Radiation, CEMI Field Theory, Wave/Particle Duality, Quantum Entanglement, Quantum Superposition, Coherence Field

\section{Consciousness and the Brain}

In 1983, philosopher Joseph Levine proposed that an explanatory gap exists between comprehension of the physical world and consciousness [1]. Matter as we know it is discrete, deterministic, tangible to the organic body, while the mind seems more indivisible, fluid, spontaneous, fleeting, closer to a holistic entity operating unobstructed by natural law than an aggregate of mechanistic parts. He asserted that this chasm between subjectivity and objectivity, anticipated during the early 20th century by Bertrand Russell in his proposed distinction of "knowledge by acquaintance" from "knowledge by description" [2], will be impossible to resolve with scientific theorizing.

Advancements in neuroscience have begun to make the problem appear more 
tractable, at least insofar as it relates to the brain. Consciousness' quality of holism resolves into the combination problem: how do trillions upon trillions of components which make up this organ interact to produce their correlates in sensation, emotion and thought? What many regard as a still greater challenge is theoretically modeling the substance of sensation, emotion and thought itself. If percepts are so strongly correlated with matter, are perception and its adjuncts an emergent material mechanism we have yet to describe, or is this field of awareness in which the brain participates incapable of being explained in terms of atomic structure? Neuroscience has recently made such great strides that it is finally possible to make firm, testable predictions about how the brain contributes to the substance of subjective experience, and this paper aims to set out what the construction of such a model entails in its essentials.

We begin with a description of how the nonsynaptic mechanisms of neurons can be understood as fluctuations in an electromagnetic field. This provides a causal link between the brain's cellular anatomy and macroscopic EM field effects such as those recorded by EEG. Evidence suggests a strong correlation between the brain's EM field and motive forces which drive consciousness, including focused attention and decision-making. CEMI field theory seems to be a preeminent hypothesis for how these multilayered EM field effects manifest as intentionality and the subconscious, so fundamentals of this framework are outlined. The quantum principles of matter and brain structure are presented, including a possible mechanism by which EM radiation emanating from regions of accelerating electric charge interacts with molecular structure to form the substance of many image and feel percepts.

\section{The Brain's Electromagnetic Field}

Introductory models of brain function usually promote the idea that ion diffusion is the mechanism by which neuronal messages are transmitted. $\mathrm{Na}^{+}, \mathrm{K}^{+}, \mathrm{Cl}^{-}$ and more flow or are ferried around neurons, causing action potentials and dendritic potentials by ion channel mechanisms. While ions are crucial for information transfer in the brain by modulating voltage gradients across membranes, the signals themselves along with cellular physiology must be accounted for primarily with reference to changes in voltage.

First, a primer on the structure of the neuron (Figure 1). In an axon, action potentials initiate at its junction with the soma, the axon hillock, traveling to the axon terminal and synaptic cleft. The axon is enveloped in insulating myelin to increase conductance speed, with relatively small, nonmyelinated segments called the nodes of Ranvier spaced at intervals along the axon. $\mathrm{K}^{+}$ions are most concentrated inside the cell, while most $\mathrm{Na}^{+}$ions are located outside the cell, maintaining gradients for outward and inward diffusion respectively [4].

Voltage-gated $\mathrm{Na}^{+}$channels are located at the nodes of Ranvier. These nodes are flanked by comparably sized paranodal regions, where myelin attaches to the neuron's cell membrane. Paranodal regions are flanked by likewise small juxtaparanodal regions containing an axon's voltage-gated $\mathrm{K}^{+}$channels. Additional 


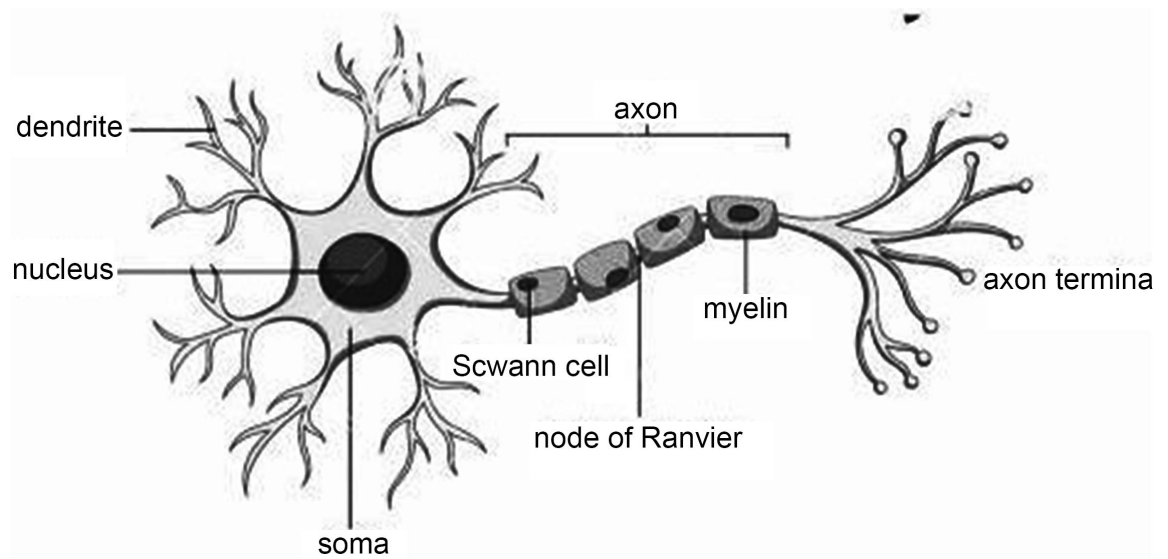

Figure 1. Structure of the neuron [3].

$\mathrm{K}^{+}$leakage channels are dispersed throughout the axonic membrane, making it highly permeable to this ion. Because the membrane is much more permeable to $\mathrm{K}^{+}$than $\mathrm{Na}^{+}$, sodium-potassium pumps help maintain functional balance by a constant ferrying of two $\mathrm{K}^{+}$ions into the cell accompanied by three $\mathrm{Na}^{+}$ions out of the cell (Figure 2) [4] [5].

At least several dendrites plus their branches are typically attached to the opposite side of the soma from the axon. Dendrites propagate signals from a synapse into the soma by also using voltage-gated $\mathrm{Na}^{+}$and $\mathrm{K}^{+}$channels, which are located both proximal and distal to the dendrite/soma junction. If EPSPs (excitatory postsynaptic potentials) caused by dendritic ion channels are strong enough, they reach the axon hillock and prompt an action potential in the axon. IPSPs (inhibitory postsynaptic potentials) are caused by influx of $\mathrm{Cl}^{-}$ions into the dendrite. These $\mathrm{Cl}^{-}$ion channels are located proximal to the dendrite/soma junctions so that less are required to mitigate incoming EPSPs. Whether dendritic potentials reach the axon hillock with enough strength to initiate an action potential is determined by a summation of the EPSPs and IPSPs of upstream dendrites [6] [7].

A model based solely on the diffusion of charge carrying ions from ion channels cannot explain why nodes are spaced closer together in larger diameter neurons even though less axial resistance-greater degrees of freedom in diffusion-should allow them to be farther apart [8]. It also fails to account for how modest increase in node width, allowing a significantly larger quantity of $\mathrm{Na}^{+}$ channels to be present, does not enhance the rate of signal transmission by way of more $\mathrm{Na}^{+}$influx and greater rates of diffusion [9]. The organization can be better described by viewing signal transmission as a lengthwise flow of electromagnetic energy driven by interactions between more positive and less positive charge. This probably occurs via a rapid chain reaction of positive/negative polarity shifts in atoms of the solution, proceeding faster from more positive to less positive when greater disparity of charge is present. Because the phenomenon, once initiated, probably involves decelerative inertia across space when charge is constant, I will provisionally name this the "ebb effect". 

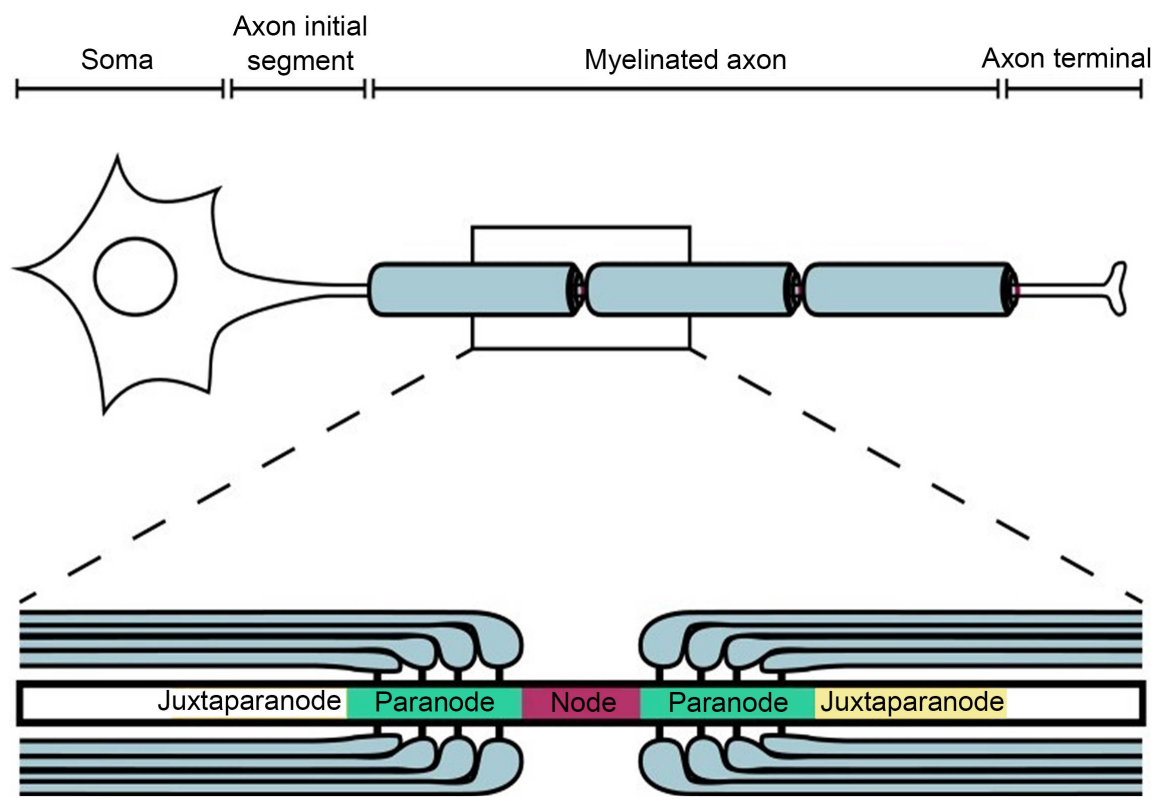

Figure 2. Nodal, paranodal and juxtaparanodal regions [4].

During an action potential, $\mathrm{Na}^{+}$ions flow into the neuron through voltagegated ion channels in the nodes of Ranvier. This depolarizes space close to the membrane, so a center of relatively positive charge is established. Voltage-gated $\mathrm{K}^{+}$channels in the juxtaparanodal regions almost immediately begin letting $\mathrm{K}^{+}$ diffuse out of the cell, making electric charge less positive in a process called hyperpolarization. This creates a strong voltage differential lengthwise along the axon and accelerates the transmitting of electromagnetic energy. At this point, juxtaparanodal regions flanking the next node of Ranvier have not fully recovered from hyperpolarization, so a much less positive center of charge remains between the first juxtaparanodal region described and the next node of Ranvier. Acceleration generated by the first lengthwise, nodal/juxtaparanodal voltage gradient carries electromagnetic energy through the internodal space until the next juxtaparanodal region's sphere of influence is reached. This much less positive locale renews acceleration of electromagnetic energy conductance so the next node of Ranvier can be depolarized, continuing the chain reaction through the length of the axon at a rate much faster than lengthwise diffusion would accomplish.

Persistent diffusion of $\mathrm{K}^{+}$back into the neuron through leakage channels and the sodium-potassium pump replenishes loss of $\mathrm{K}^{+}$, while the sodium-potassium pump prevents the cell from being overloaded with $\mathrm{Na}^{+}$. This lengthwise voltage gradient mechanism is similar in dendrites despite a lack of myelination, with voltage-gated $\mathrm{Na}^{+}$channels concentrated at particular locations to create strong centers of positive charge. $\mathrm{Cl}^{-}$ions act as a negative attractor at the base of dendrites. High concentrations of $\mathrm{Cl}^{-}$can mitigate an EPSP such that its electromagnetic energy carried by positive charges does not attain enough strength to transit the soma and reach the axon, while extremely low concentrations would be insufficient to function as a negative attractor, so $\mathrm{Cl}^{-}$concentrations must al- 
so be regulated to median levels. Axon hillocks have the largest concentration of voltage-gated $\mathrm{Na}^{+}$channels in a neuron because the positive charge must be strong enough to overcome attenuation by reverse propagation into the adjacent soma along with a greater degree of repolarization in the closest juxtaparanodal regions due to their location farther upstream within the action potential chain.

Flow of ions in and around neurons generates steady state electromagnetic fields measured with an electrode as LFPs (local field potentials). If all signal transmission in the brain is electromagnetic energy flow driven by voltage gradients, communication between neurons must involve constant fluctuations in these EM fields, as evidence corroborates. Analysis shows that $\mathrm{Na}^{+}$influx causes rapid and short perturbation, widespread $\mathrm{K}^{+}$diffusion is characterized by more prolonged perturbations of lower intensity, and the somewhat less nodal structure of unmyelinated dendrites results in LFP perturbations that generally decay slower with time [10]. On the scale of ion channels, magnetic effects are significant, but as brain structure ascends upward in scale, magnetism quickly becomes negligible and the field is primarily electric. LFPs interact to form emergent flow shapes, and expansive neural networks comprise still different shapes of even more emergence, culminating in the organwide electric field flows registered by EEG [11].

If brain function is so closely associated with electric field properties, and these properties take effect on a macroscopic, even global scale, this suggests obvious parallels to the ultraintegrated, fluid holism of consciousness, our minds perhaps being an emergence of field-related mechanisms. Does consciousness correspond in some way to the brain's EM field?

\section{Large-Scale Mechanisms of the Brain's EM Field}

Linkage between wave oscillations of the brain's electric field and awareness is well-documented. Delta waves oscillating at EEG frequency $0.5-3 \mathrm{~Hz}$ occur during sleep. Theta waves $(3-8 \mathrm{~Hz})$ show up while in a daydreaming state between sleep and wakefulness. Alpha waves $(8-12 \mathrm{~Hz})$ are associated with a relaxed, idling state of mind such as when we pause with our eyes closed. Beta waves $(12-38 \mathrm{~Hz})$ happen during alert states of intellectual activity and outwardly focused concentration. Gamma waves $(38-42 \mathrm{~Hz}$ ) arise in conjunction with many neocortical contributions to perception and consciousness, such as analytical problem-solving [12].

Some wave types are strongly tied to certain regions of the brain. The hippocampus involves theta activity, the motor cortex features beta activity, and as was mentioned, gamma activity can obtain in the neocortex. Traveling waves of various frequencies traverse paths through the electric field ranging from a few millimeters to dozens of centimeters, and have been observed spanning the entire neocortex. It is noted that the strongest traveling waves incline to be out of phase with the rest of the brain [11]. If tied to high arousal consciousness, this explains why fully attentive states consist in serial processing as opposed to the massively parallel processing of unconscious states. We might be able to intentionally con- 
centrate on only a limited range of tasks because the electric fields of alert, focused consciousness segregate more from what surrounds them.

A typical explanation for large-scale electric field flows is that neural networks are synchronized by feedback loops, similar in concept to central pattern generators but so tightly coupled in recurrence that the emergent electric field evinces an in-phase pattern of oscillation as it moves. Experiments with electrodes inserted into in vitro nervous tissue have suggested that neurons engage in a phase locking mechanism which is still poorly understood, allowing the cells to fire in perfect, in-phase synchronicity. Researchers suspect that this phase locking is mediated by interaction of EM fields with the molecular structure of ion channels [13]. Phase locking between ion channels and the EM field would certainly have pervasive effects, but it is plausible that much additional biochemistry could synchronize into EM fields due to complementary electromagnetic properties. Atoms are like tiny magnets, and even complex molecules may be sensitive to the motions of supervenient electric fields. Perhaps electric currents can almost causally saturate some tissues of the brain as they oscillate and flow.

Molecular biologist Johnjoe McFadden has proposed CEMI (conscious electromagnetic information) field theory, which claims the brain's EM field is a motive force driving the activity of neural networks, and when these effects are strong enough they give rise to CEMI fields responsible for the causality and experience of willed agency. Some neurons have adapted for sensitivity to EM fields, and these are implicated in conscious brain processes, allowing us to control our attention and make decisions, while EM field insensitive neurons participate in unconscious processes. He explicitly asserts that the disjunction of CEMI fields from bordering EM fields can explain distinctly serial processing of consciousness [13].

It is the current author's opinion that three factors must determine whether an EM field graduates to something like CEMI field status, becoming intentional will. Molecular structure of the tissues involved must be such that they are acutely responsive to EM field flows. The domain of the EM field must be large enough to incorporate holistically functional regions within its sphere of action. And EM field effects must be densely concentrated enough within tissue that an intensity threshold is surpassed. If EM fields minimally interact with tissue, are dispersed or remain small-scale, they may evoke lower arousal subconscious processes but will not enter into peak consciousness [14].

The plethora of evidence for electric field to awareness correlation alongside confirmation of feedback loop integration and phase locking mechanisms makes it seem as if neuroscience is well on its way to resolving the combination problem insofar as it relates to functional coordination. EM fields are not only a signature of neural network synchronicity but so far appear to actively modify activity throughout the brain, conjuring both low and high arousal states within large swaths of tissue. If CEMI fields are proven to exist with conclusiveness, this easily explains how intentional will manifests as structurally unified and causally efficacious. But though the forces which drive neural network synchronicity may 
be demystified by research along these lines, it is still not apparent why so-called will, ranging from the most unconscious to the most conscious processes, looks or feels like anything. What are the brain mechanisms that contribute to the substance of percepts and perception?

\section{The Substance of Perception as a Consequence of Interactions between Electromagnetic Radiation and Biochemistry}

The most obvious and well-understood example of what it means to look or feel like something is a color percept, so we can begin to unravel subjectivity with a basic analysis of light. Electromagnetic radiation travels through the environment as a field with specific wavelengths, spreading in all directions. These radiating waves can be absorbed and emitted by electromagnetic matter in discrete packets or quanta called photons, so they have both wave and particle properties, a phenomenon referred to as wave/particle duality. Whether EM radiation is absorbed or emitted by an atom as a photon is determined by its wavelength and corresponding frequency or energy. As unabsorbed electromagnetic radiation flows through highly permeable portions of the environment such as Earth's atmosphere or the vacuum of outer space at a breakneck speed of around 300 million meters per second, its wavelengths blend to produce combinations. This property of multifaceted yet simultaneous and hybridized structure is called quantum superposition. EM radiation also interacts through nonlocal mechanisms that remain mostly unknown, so perturbing a photon as it travels correlates statistically with changes to photons of common chemical origin moving in alternate directions. This is called quantum entanglement, an interaction that appears to occur faster than light speed, and which has even been observed as retroactive in cunning experiments.

The human eye is sensitive to EM radiation of wavelength $400-700 \mathrm{~nm}$ : the visible spectrum from violet to red. Light waves in this range are absorbed as photons by photoreceptor cells in the retina where they perturb molecular structures. Biochemical pathways convert these molecular perturbations into a neuronal signal which travels through the optic nerve to the brain by voltage dynamics described above, eventually arriving at the visual cortex in the occipital lobe for processing into a perceptual image (Figure 3).

Neural processing then rapidly makes its way from the back of the head to more anterior regions of the brain, adding layers of successively greater generalization to the perceptual field, such as a color palettes, shapes and relative sizes. The dorsal pathway trajecting towards the parietal lobe processes "where and how" features as increasingly inclusive data related to position and motion. It culminates adjacent to motor regions near the top of the brain that are the keystone of voluntary movement. The ventral pathway trajects into the temporal lobe and processes "what" features such as object and facial recognitions. Predominantly grey matter (dendrites and soma) of the separated dorsal and ventral pathways coordinates via interposed white matter which is an integrating web of 


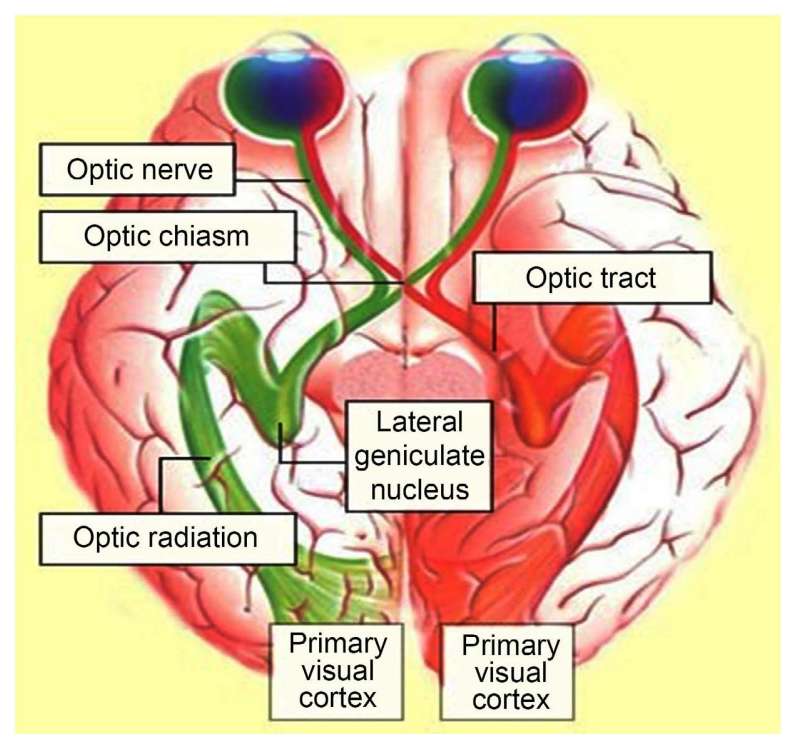

Figure 3. Pathway from the eye to the visual cortex [15].

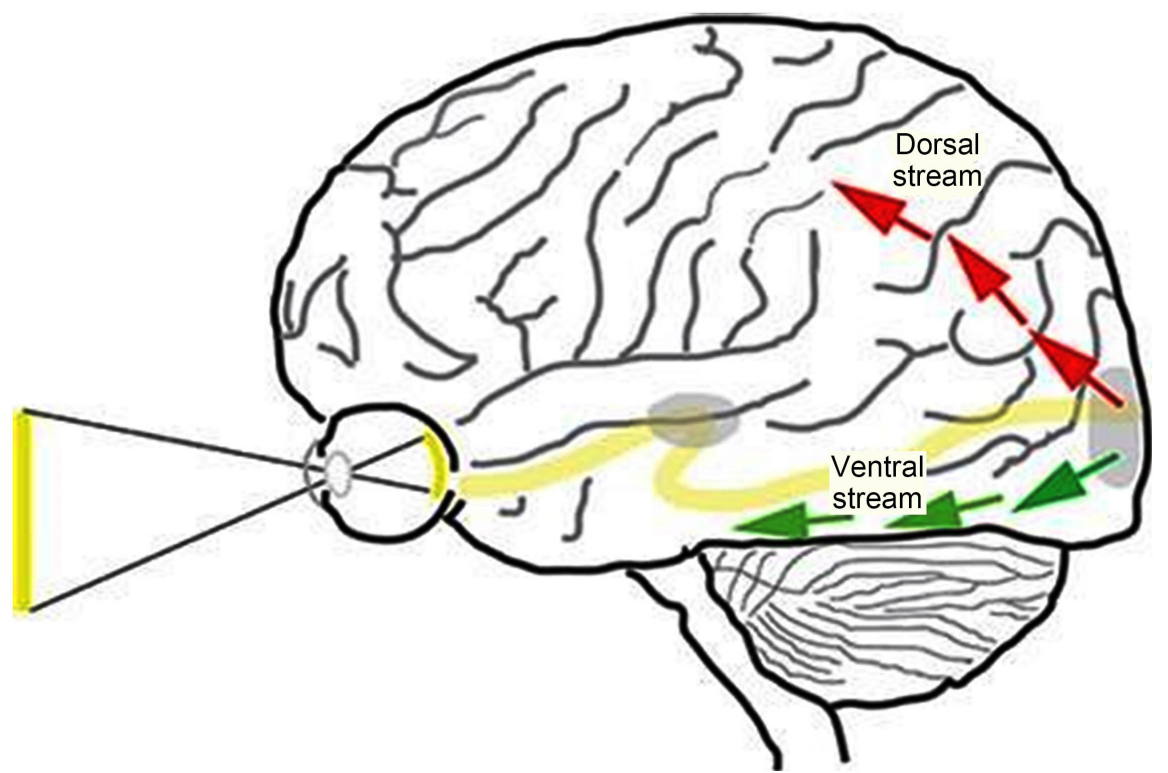

Figure 4. Dorsal and ventral pathways of the visual system [16].

axonic connections that run both ways, helping the entire visual system to function as a cohesive unit (Figure 4).

Almost all properties of visual perception can currently be identified in terms of neural structure except the most interesting part: why are the subjective phenomena that correlate with electrical signals a percept and not merely an electrical signal? What is it about reality and the way our brains interact with it, whether constituted of matter or something else, that contributes towards making perception a distinct property from conventional anatomy?

It is not unfruitful to speculate that biochemical properties can, in consort with EM radiation, be largely sufficient to produce color percepts, because these forms of matter-atoms and photons-are not as distinguishable in their prin- 
ciples of action as a casual glance might lead one to assume. The double-slit experiment has created interference patterns from emission of molecules as large as two thousand atoms, so wave/particle duality applies at a much larger scale than photons and electrons [17]. These effects are harder to induce as mass increases, so it seems that bigger size skews molecules towards the particle end of the structural spectrum. Superposition also occurs within molecules, but to a more limited extent than in light waves. The hydrogen atoms of methane $\left(\mathrm{CH}_{4}\right)$ have been shown to superposition with the central carbon atom, overlapping in intermediate space [18]. A tentative conclusion might be that hydrogen's bonds extensively superposition in nature. But the evidence so far indicates that as molecules increase in size, their atoms become more particlelike and are less prone to overlap in superposition states. 15 million atoms have been entangled at once, an experiment performed on a gaseous mixture at the unprecedentedly hot temperature of $176.9^{\circ} \mathrm{C}$. Entanglement was originally only achievable with supercooled chemicals, but has subsequently worked at room temperature [19]. Researchers even managed to entangle two aluminum drums of 1 trillion atoms each, about the size of red blood cells, which synchronously vibrated by the diameter of one proton at temperatures near absolute zero [20]. It seems all sorts of conditions are conducive to entanglement between relatively large conglomerations of atoms, but again the effects have been harder to attain in the lab with structures that are chemically bonded in more large-scale or complex ways.

Difference between the extreme wave, superposition, entanglement behavior of light and the generally more constrained behavior of larger masses is attributed to decoherence. As mass increases, more particles are jostling entropically in a process that tends to cause them to interfere, canceling out their ability to spread and interact across relatively large space so that they become more localized [21]. The opposite of decoherence is termed coherence, a state in which wave, superposition and entanglement properties can broadly apply.

Whether decoherence happens is determined by entropy, the disorder in a material system, and chemical structure rather than mass per se, though entropic factors such as thermal energy can of course limit the ability of mass to form large chemical structures, hence the rather loose correlation between mass and decoherence. Relatively low entropy chemical structures of large mass can give rise to coherent states if conditions are suitable, and somewhat higher entropy matter of smaller mass can as well give amenable chemical structure.

The following are some illustrations of the relationship between decoherence and coherence. An electron hurtles through the double-slit experiment at 6 million meters per second, a highly entropic state allowing a single photon to disrupt the electron's path and prevent an interference pattern from materializing on the screen at the back of the vacuum chamber. A copper wire is comparatively nonentropic, its atoms fixed in place as an extremely stable solid, supporting the flow of constituent electrons as a rapid coherence current when electricity is applied. Saline solution is more entropic, its water molecules, sodium ions and chloride ions engaged in jostling diffusion over such large spaces per unit of time 
that they bond in chemical structures no larger in diameter than an ion's nanoscale solvation shell. But when electricity is applied, positive and negative charges act as a bridge, perhaps by a similar but opposite mechanism to electrical transmission in neurons, allowing electromagnetic energy to move at a rapid enough rate that the solution is relatively stationary over short timespans, almost as if a quasimetal. This is a strong coherence current, but less rapid and far-reaching than in copper wires. Raising ion concentration increases the quantity of emergent solvation shell structures, which can lower average entropy of the solution so electrical coherence is transmitted more forcefully. A large organic molecule interacts with surrounding solution such that a lot of decoherence happens at its fringes, but inner portions probably remain low enough in entropy for some kind of coherence to be in effect, at least to the extent permitted by a residual jostling of chemically bonded atoms, though how exactly this might work remains unknown. Organic molecules are often fixed in place by cellular structures like cytoskeletal fibers and membranes, reducing entropy and in theory facilitating even more coherence.

The dynamics of macroscopic particles are driven by thermal energy and involve a substantial degree of decoherence, dividing classical from quantum phenomena, but within a multitude of conditions at the atomic scale coherence still readily takes effect. Like light, individual atoms and even fairly large atomic and molecular structures have wavelength, superposition and entangle. So while only EM radiation behaves like a textbook wave, it is possible to regard atomic structure at the microscale as comprised of wavicles which can share in all the essential coherence properties of light. It makes sense then to consider the possibility that wavicles not only form chemical bonds and functional structures in relation to each other as well as absorb or emit light, but can cohere with EM radiation in a complex of atomic nodes within photonic fields.

The speed of light is effectively instantaneous in the brain, perhaps capable of synchronizing with numerous molecules in a simultaneous way by entanglement, and blending into atomic structure as superposition, far beyond the intricacy of EM radiation alone. Acceleration of electric currents generates EM radiation of lower frequency than that which is fully absorbed into or reflected by atoms, and this may be the primary substrate of superposition fields. In nervous systems and brains, one of the most likely locations for this low frequency light emission is between the nodes of Ranvier and juxtaparanodal regions, where electromagnetic energy flow is accelerated by reduced concentration of $\mathrm{K}^{+}$. An even more probable candidate is the dendrites, where electromagnetic energy accelerates over relatively long distances, moving between clustered sodium channels in distal regions and the negative, $\mathrm{Cl}^{-}$centers of charge around the dendrite/soma junction. The presence of multiple dendrites increases the amount of radiation, perhaps to levels that can interact with neural chemistry on a broad scale, conceivably even breaching the soma. If sites of current cause trillions upon trillions of radiative locations in stretches of neuron, and radiative fields travel in all available directions across the distance of many micrometers, this 
may be enough to bind hundreds if not thousands of molecular structures into individual units pervading the brain. Subjective color might consist in superpositions between EM radiative fields and biochemistry, an ultrahybrid resonance of waves and wavicles, obeying the same structural principles of quantum physics as both atoms and free flowing light in environments outside the body. Image percepts could be constructed from these units of resonance.

Of course this theory of resonance is full of uncertainties, just as the study of neural networks was decades ago. A way to verify and then study likely resonance properties is required, enlisting physics in the development of neuroscience to contrive new observational methods and models. But this seems the most probable hypothesis for image perception yet. The simplest possible explanation is that the visual field is an analogue to quantum effects operative upon light and atoms separately. Strong circumstantial evidence comes from the way this accounts for how brain matter has a darkish tint while myelin is white. The grey matter of dendrites, some and the interior of axons is darkly shaded to absorb large amounts of EM radiation, while myelinated white matter reflects as much as possible so that radiative fields minimally attenuate across space. From the outside, neural matter looks greyish, but from the inside it synchronizes via entanglement and blends in superposition to bind as the substance of image perception.

It stretches the imagination when we try to think about how organization of matter in the brain can give rise to this perceptual field which appears so convincingly to be outside of the body. We must remember that the sharply focused visual field is only as large as the size of your thumb held at arm's length in front of the face, with the majority of human vision pieced together from disjointed segmentations of eye saccading and even moreso involuntary memory functions. The visual field is mostly assembled by the brain from patches of stimulus that are fractional in space and temporally separated, so regardless of how physiology has adapted for experiencing, the mechanisms of our perception are largely within the brain. The way resonances hypothetically give rise to the substance of image percepts is really not a different issue in its fundamentals from how neural circuitry coordinates the cells within which these percepts would reside. Just as neural networks can be mapped according to their functional units, percept units could map onto the molecular structure of neurons insofar as it coheres with EM radiation. If this hypothesis is proven accurate, all kinds of new molecular functions and perhaps novel classes of functional molecule will be found, with heavy reliance on quantum physics in constructing these models. Ongoing discovery of the feedback loops associated with neural circuitry alongside progressing models of EM field synchronization effects as discussed above can probably explain how these radiative/molecular percepts are orchestrated to form an intelligible picture of the world around us. In addition to vision, this new theory can potentially model mental images and hallucinatory artifacts of brain processes, perceptual phenomena which are not derived from direct stimulation by the en- 
vironment.

This theory would easily verify the mechanism of how percepts look, but what about how they feel? Why does perception have these nondimensional qualities in addition to spatial extension and temporal duration? When we think about resonance, the most characteristic property is vibration. All matter from the atomic to the macroscopic scale vibrates, and it is difficult to come up with a vibration that does not feel like something. Stretching and flexing of our skin, the bending of our eardrum, the soft or harsh glow of light with its frequencies and wavelengths, it all feels like something. The following is a tentative suggestion still to be proven by solid evidence, but perhaps it is intrinsic of waves and wavicles to consist in fragments of feeling as they resonate both independently and jointly. However, matter on the nanoscale does not seem to feel with much resolution. Specially adapted structures apparently exist throughout the body that increase the resolution of these resonances by idiosyncratic and intricate chemical organization, resulting in emergently complex feelings of incalculable diversity. If resonance between atoms and EM radiation produces perceptual feel as well, the variety in possible mechanisms is effectively limitless when we consider all the structural forms conceivable. The basics of feeling as molecular structures bound into percepts by EM radiation may constitute an even larger spectrum than image percepts.

Before the maturation of neuroscience, most philosophers and scientists viewed the substance of consciousness and its interactions with the environment as mediated by a nonphysical field. Nonlocal entanglement between matter separated by hundreds of kilometers certainly defies a model based on brain properties alone. But insofar as perception is tied to the brain, it seems promising that a theory of electromagnetic matter might be largely sufficient to describe many percepts along with motive forces that drive behavior of this matter on the electromagnetic scale. If an underlying, even nonelectromagnetic field with extremely remote effects is unveiled, it obviously must interact with these molecular and radiative quanta to impact the brain. Wave/particle duality, superposition and entanglement will most likely be core to any theory of consciousness regardless of how exotic our knowledge of physical force becomes.

\section{The Quest for a Theory of Consciousness and the Brain}

Preliminaries of a complete explanation for the brain's role in consciousness seem available to us. In neurons, a chain reaction of electromagnetic energy rapidly travels from more positive to less positive centers of charge, accelerated by periodically increasing charge disparity between neural regions. Feedback loops amongst neurons as well as phase locking between EM fields and neuronal chemistry synchronize these electromagnetic flows within large swaths of brain tissue, the emergent shapes of oscillating and traveling waves as recorded by EEG. A plausible hypothesis is that the electromagnetic causality imposed by these large-scale waves is responsible for the subconscious, intentional consciousness, 
and the experience of our own willed agency. Acceleration of electromagnetic energy in many trillions of locations within neurons of the brain emits EM radiation, and these radiative waves may interact with molecular wavicles by way of shared quantum mechanisms such as superposition and entanglement to bind biochemistry into percepts. Wave/wavicle resonances might not only comprise complex subjective images, but vibrations involved could include the basic constituents of feeling. These dimensional and/or nondimensional structures would form emergent arrays and conglomerations that are specialized to increase resolution, and together with EM field synchronization project the perceptual field insofar as it arises from the brain.

Percepts may at base be a product of quantum coherence properties and mechanisms, so if justified by subsequent evidence, this entire apparatus of electromagnetic energy flow, feedback loop or EM field synchronization, and wave/ wavicle binding can be termed a "coherence field". Whatever nonlocal fields and forces cause the extremely remote causality that is fast being revealed by experimentation in physics, it would of necessity interact with electromagnetically quantum phenomena, so this entire edifice of physical knowledge, at least to the extent that it intersects with consciousness and especially the brain, might be subsumable under the heading of coherence field theory. If coherence fields are proven to exist as outlined above, this will set science on course to resolve the explanatory gap and bring knowledge by acquaintance a great deal closer to knowledge by description.

\section{Conflicts of Interest}

The author declares no conflicts of interest regarding the publication of this paper.

\section{References}

[1] Levine, J. (1983) Materialism and Qualia: The Explanatory Gap. Pacific Philosophical Quarterly, 64, 354-361. https://doi.org/10.1111/j.1468-0114.1983.tb00207.x

[2] Russell, B. (1910) Knowledge by Acquaintance and Knowledge by Description. Proceedings of the Aristotelian Society, 11, 108-128.

https://doi.org/10.1093/aristotelian/11.1.108

[3] Youtube.com. Labeled Diagram of Neuron. https://www.youtube.com/watch? $\mathrm{v}=$ surKjBAs8I8\&t=0s

[4] Arancibia-Carcamo, L. and Attwell, D. (2014) The Node of Ranvier in CNS Pathology. Acta Neuropathologica, 128, 161-175. https://doi.org/10.1007/s00401-014-1305-Z

[5] Humphries, E.S.A. and Dart, C. (2015) Neuronal and Cardiovascular Potassium Channels as Therapeutic Drug Targets. Journal of Biomolecular Screening, 20, 1055 1073. https://doi.org/10.1177/1087057115601677

[6] Hanson, J.E., Smith, Y. and Dieter, J. (2004) Sodium Channels and Dendritic Spike Initiation at Excitatory Synapses in Globus Pallidus Neurons. Journal of Neuroscience, 24, 329-340. https://doi.org/10.1523/JNEUROSCI.3937-03.2004

[7] Ames, H., Gorchetchnikov, A., Leveille, J. and Versace, M. (2008) KinNeSS: A Mod- 
ular Framework for Computational Neuroscience. Neuroinformatics, 6, 291-309. https://doi.org/10.1007/s12021-008-9021-2

[8] Ford, M.C., Alexandrova, O., Cossell, L., Stange-Marten, A., Sinclair, J., KoppScheinpflug, C., Pecka, M., Attwell, D. and Grothe, B. (2015) Tuning of Ranvier Node and Internode Properties in Myelinated Axons to Adjust Action Potential Timing. Nature Communications, 6, Article No. 8073. https://doi.org/10.1038/ncomms9073

[9] Arancibia-Cárcamo, I.L., Ford, M.C., Cossell, L., Ishida, K., Tohyama, K. and Attwell, D. (2017) Node of Ranvier Length as a Potential Regulator of Myelinated Axon Conduction Speed. eLife, 6, Article ID: e23329. https://doi.org/10.7554/eLife.23329

[10] Bedard, C., Kroger, H. and Destexhe, A. (2004) Modeling Extracellular Field Potentials and the Frequency-Filtering Properties of Extracellular Space. Biophysical Journal, 86, 1829-1842. https://doi.org/10.1016/S0006-3495(04)74250-2

[11] Alekseichuk, I., Flachier, A.Y., Linn, G., Xu, T., Milham, M.P., Schroeder, C.E. and Opitz, A. (2019) Electric Field Dynamics in the Brain during Multi-Electrode Transcranial Electric Stimulation. Nature Communications, 10, Article No. 2573. https://doi.org/10.1038/s41467-019-10581-7

[12] Sinha Clinic (n.d.) What Are Brainwaves? https://www.sinhaclinic.com/what-are-brainwaves/

[13] McFadden, J. (2021) The Electromagnetic Will. NeuroSci, 2, 291-304. https://doi.org/10.3390/neurosci2030021

[14] McFadden, J. (2013) The CEMI Field Theory Gestalt Information and the Meaning of Meaning. Journal of Consciousness Studies, 20, 1-2.

[15] The Brain from Top to Bottom, Vision (n.d.). https://thebrain.mcgill.ca/flash/d/d $02 / \mathrm{d} 02 \mathrm{cr} / \mathrm{d} 02 \mathrm{cr}$ vis $/ \mathrm{d} 02 \mathrm{cr}$ vis.html

[16] Sheth, B.R. and Young, R. (2016) Two Visual Pathways in Primates Based on Sampling in Space: Exploitation and Exploration of Visual Information. Frontiers in Integrative Neuroscience, 10, Article No. 37. https://doi.org/10.3389/fnint.2016.00037

[17] Letzter, R. (2019, October 4) 2,000 Atoms Exist in Two Places at Once in Unprecedented Quantum Experiment.

https://www.livescience.com/2000-atoms-in-two-places-at-once.html

[18] McFadden, J. (2020) Integrating Information in the Brain's EM Field: The CEMI Field Theory of Consciousness. Neuroscience of Consciousness, 1, Article No. niaa016. https://doi.org/10.1093/nc/niaa016

[19] Irving, M. (2020, May 17) 15 Trillion Quantum Entangled Atoms Make a RecordBreaking Hot Mess.

https://newatlas.com/physics/15-trillion-atoms-quantum-entanglement/

[20] Garisto, D. (2021, May 6) Scientists Supersize Quantum Effects with Entangled Drum Duet. Scientific American.

https://www.scientificamerican.com/article/scientists-supersize-quantum-effects-wi th-entangled-drum-duet1/

[21] McFadden, J. (2014) Life on the Edge: The Coming of Age of Quantum Biology. Crown Publishers, New York. 